\title{
Video-análisis de una actividad de indagación en Primaria
}

\author{
Ana Aragüés Díaz. IES Valle del Ebro \\ Recepción: 26.10.2019 | Aceptado: 30.10.2019 \\ Correspondencia a través de ORCID: Ana Aragüés \\ iD 0000-0002-8228-0813 \\ Aragüés, A. (2019). Video-análisis de una actividad de indagación en Primaria. REIDOCREA, 8, 309-318.
}

\begin{abstract}
Resumen: Esta investigación se centra en descifrar lo que acontece en un aula cuando una futura maestra (Practicum Universitario) trata de desarrollar una actividad de ciencias basada en la indagación con alumnos de $6^{\circ}$ de Primaria (11-12 años). Para ello, empleando la técnica del video-análisis, se han examinado varias escalas de análisis como son: 1) aspectos generales de la sesión 2) el tipo de estructura realizada por la futura maestra en términos de juegos didácticos (Teoría de la Acción Didáctica Conjunta; Sensevy, 2011) para llevar a cabo la actividad, así como 3) el tipo de estrategias de indagación percibidas en el alumnado de Primaria. Los resultados muestran cómo una estructuración basada en preguntas por parte de la futura maestra facilita un acercamiento al proceso de indagación al alumnado de Primaria. Se percibe cómo al final de la actividad son incluso los estudiantes los que llegan a plantear preguntas susceptibles de ser debatidas. También son observadas ciertas dificultades cuando los alumnos tratan de relacionar los datos obtenidos en la sesión con sus marcos teóricos previos.
\end{abstract}

Palabras clave: Educación Básica I Enseñanza de las Ciencias

\section{Video-analysis of an inquiry activity in Primary}

\begin{abstract}
This research focuses on deciphering what happens in a classroom when a future teacher (University Practicum) tries to develop a science activity based on inquiry with 6 th grade students (11-12 years old). To do this, using the video-analysis technique, several scales of analysis have been examined such as: 1) general aspects of the session 2) the type of structure made by the future teacher in terms of didactic games (Joint Didactic Action Theory; Sensevy, 2011) to carry out the activity as well as 3 ) the type of inquiry strategies perceived in Primary students. The results show how a structuring based on questions by the future teacher facilitates an approach to the process of inquiry of Primary students. It is perceived how at the end of the activity even the students come to ask questions that can be debated. Certain difficulties are also observed when students try to relate the data obtained in the session with their previous theoretical frameworks.
\end{abstract}

Keywords: Elementary Education I Science Education

\section{Introducción}

Los primeros momentos en los que un docente se enfrenta por primera vez a una clase, se caracterizan por la aparición de una serie de dificultades que nacen de la transposición de la teoría a la práctica (Terigi y Diker, 1997). No es lo mismo lo que un docente dice que va a hacer y lo que verdaderamente acaba haciendo en clase. Sobre esta idea, en este estudio nos hemos enfocado en obtener una comprensión profunda sobre qué es lo que acontece en el aula cuando se trata de trabajar mediante indagación en clases de ciencias de Primaria (Crujeiras Pérez y Jiménez Aleixandre, 2015).

Trabajos como los de Rivero García, Solís Ramírez, Porlán Ariza, Azcárate Goded y Martín del Pozo (2017) señalan que los maestros en formación inicial suelen poseer un enfoque de la educación principalmente de tipo transmisivo, por lo que no es de extrañar que investigaciones como las de Bikner-Ahsbahs, Monaghan, Radford y Sensevy (2012) muestren la difícil integración que supone la indagación en el aula. La indagación, como una forma de aprendizaje de las ciencias se ha convertido en una 
constante en los actuales trabajos de investigación en lo que a didáctica se refiere (Crujeiras-Pérez y Cambeiro, 2018; Solé Lussà, Aguilar Camaño, Ibañez y Coiduras Rodriguez, 2018; Martínez Chico, López-Gay y Jiménez Liso, 2014). Pero ¿qué se entiende por indagar? Hace tres décadas, investigaciones como las de Driver, Guesne y Tiberghien (1985) favorecieron a la creación de un cuerpo de conocimientos que pone de manifiesto cómo los alumnos tienen sus propias ideas con cierto carácter universal, aunque éstas no siempre coincidan con las aprobadas científicamente. Estos resultados tuvieron una gran relevancia para lo que se denominó el modelo de cambio conceptual (Duschl, 1995), propuesto como una forma de transformación de esas preconcepciones del alumnado. Este modelo expresa la necesidad de un cambio en el tipo de guía docente durante el aprendizaje. Podría decirse que el sentido de la indagación en el aula recoge la idea esencial del modelo de cambio conceptual añadiendo además un fuerte componente epistemológico. Este componente enfatiza sobre la idea de un aprendizaje de la ciencia basada en su praxis, si no bien como una auténtica investigación, al menos, de una forma adaptada. Por tanto, indagar en la escuela supone no sólo aprender un contenido sino también la forma en la que éste se construye, equiparando así el saber con al saber hacer en el aula (Gil Pérez, 1991). Podemos asumir que si bien aprender ciencia en el aula no es del todo comparable a cómo la ciencia se construye, al menos es en gran medida una guía de los alumnos hacia la realización de prácticas epistémicas del trabajo científico (Vílchez González y Bravo Torija, 2015; National Research Council, 2007).

En un intento por acotar lo que el término indagación realmente implica en un contexto escolar, la literatura hace referencia a una gran variedad de subprocesos como son la resolución de problemas, la identificación de preguntas, la formulación de hipótesis, el diseño de experimentos, la toma y el análisis de datos, un uso apropiado de herramientas y técnicas o la comunicación de conclusiones (Abd-El-Khalick y Akerson, 2004). El conocimiento pasa así a emerger del diálogo en el aula, siendo el uso de pruebas una fuente principal para su desarrollo.

Cabría aclarar que la indagación en el aula tampoco debe interpretarse como un proceso fundado exclusivamente en la evidencia, en la medida que en la ciencia no todo el conocimiento científico es resultado de un proceso empírico (Kuhn, 1986). Hacer ciencia en la escuela es el resultado de una transposición directa de lo que supone una actividad científica, de manera que hablar de indagación supone más bien hablar de estrategias a resolución a problemas. En este sentido, si lo que queremos es medir en qué medida los alumnos son capaces de indagar es imprescindible el empleo de un instrumento que recoja una serie de estrategias definidas de forma clara, concisa y adaptada a la etapa escolar. Para ello nos hemos basado en el trabajo de Milne (2008) centrado en el estudio del desarrollo de habilidades de indagación en niños de Primaria. Se trata de un total de ocho estrategias de indagación (Tabla 1). Estas estrategias guardan una clara conexión con los subprocesos desarrollados por Abd-El-Khalick y Akerson (2004) aunque de una forma adaptada a la etapa de Primaria.

\begin{tabular}{|l|l|}
\hline \multicolumn{2}{|c|}{ Tabla 1. Estrategias de indagación para la etapa de Primaria. } \\
\hline \multicolumn{2}{|c|}{ Estrategias de indagación } \\
\hline (1) Explorar & Sobre un problema, situación, fenómeno, artefacto, modelo, acontecimiento, historia... \\
\hline (2) Observar & El fenómeno que se va a tratar de forma global. \\
\hline (3) Identificar evidencias & Selección de partes del dispositivo experimental. \\
& Aspectos clave que tratar. \\
& Cambios que han tenido lugar. \\
& Partes implicadas. \\
& Mediante la selección de registros y toma de datos relevantes. \\
\hline (4) Buscar explicaciones & Qué es lo que hacen estas partes/estructuras. \\
& Cuál es la causa y el efecto de los cambios. \\
& Cuál es la función. \\
\hline
\end{tabular}




\begin{tabular}{|l|l|}
\hline & $\begin{array}{l}\text { Qué partes están interactuando. } \\
\text { Cuál es el resultado de esa interacción. } \\
\text { Que tendencias y pautas tienen lugar. } \\
\text { Creación de razones personales basadas en la evidencia (en caso de que haya). }\end{array}$ \\
\hline $\begin{array}{l}\text { (5) Comprobar las explicaciones/ } \\
\text { Evaluar la explicación }\end{array}$ & $\begin{array}{l}\text { Introducción de nuevas variables. } \\
\text { Planificación de procesos para probarlas: Medir, comparar, verificar, probar, identificar, clarificar. } \\
\text { La autoevaluación de estas investigaciones puede conducir a nuevas o modificadas explicaciones, } \\
\text { dudas sobre ideas previas o conclusiones provisionales. Estas explicaciones provisionales se } \\
\text { comunican a los compañeros para la evaluación de grupo y reestructuración. }\end{array}$ \\
\hline $\begin{array}{l}\text { (6) Sintetizar lo aprendido mediante } \\
\text { producciones orales, pictóricas }\end{array}$ & $\begin{array}{l}\text { Importancia de la indagación como una comunicación del conocimiento. En primaria es de vital } \\
\text { importancia reconocer las ideas claves. Ayuda a relacionar el proceso de construcción de } \\
\text { conocimiento con el conocimiento. } \\
\text { En ciencia este paso no es necesario, pero en el aula en importante tenerlo en cuenta. }\end{array}$ \\
\hline $\begin{array}{l}\text { (7) Predecir utilizando el } \\
\text { conocimiento/ Más investigaciones }\end{array}$ & Utilización del conocimiento para predecir futuras e hipotéticas situaciones. \\
\hline (8) Aplicación a otros contextos & Búsqueda de más explicaciones, posteriores investigaciones. \\
\hline
\end{tabular}

La estrategia Explorar (1) hace referencia a una toma de contacto inicial que el alumno realiza con el objeto de estudio. Observar (2) designa la situación en la que el alumno observa el proceso o fenómeno de manera global, que a diferencia de la estrategia Explorar (1) implica un cierto grado de atención. Identifica evidencias (3) se relaciona con la observación de cambios producidos una o más partes del fenómeno, así como la toma de registros o de datos. Buscar explicaciones (4), se refiere a cuando los estudiantes establecen relaciones entre partes del sistema objeto de estudio de forma causal, funcional, de integración o establecen posibles pautas. La Comprobación de las explicaciones/ Evaluación de la explicación (5) surge cuando las explicaciones son comunicadas al grupo y sometidas a evaluación en la medida que puedan ser contrastadas, comparadas o razonadas en base a lo que se conoce. La estrategia de Síntesis de lo aprendido mediante producciones orales, pictóricas (6) hace referencia a cuando los alumnos elaboran resúmenes, ya sea de forma oral o pictórica, que den cuenta del camino que les ha conducido a elaborar las conclusiones. La estrategia Predice utilizando el conocimiento/ Más investigación (7) se refiere a cuando los alumnos utilizan una serie de contenidos científicos que han sido trabajados para establecer relaciones que les permitan realizar predicciones sobre una hipotética o futura situación (a diferencia de lo que supondría una hipótesis, la cual puede ser elaborada de forma azarosa). La última estrategia, Aplicación a otros contextos (8), es identificada cuando los alumnos gestionan el conocimiento tratado en el aula aplicándolo en un contexto distinto del que fue elaborado. Es importante destacar que el desarrollo de estas estrategias no responde a una secuencia lineal sino que se trata más bien de un proceso cíclico de estrategias resultado de una búsqueda constante explicaciones (Windschitl, Thompson y Braaten, 2008).

Una vez definidas el conjunto de estrategias de indagación para la etapa de Primaria, encontramos la necesidad de la utilización de un marco teórico social y antropológico que nos permita el estudio de las acciones que acontecen dentro del aula. Este marco lo hemos encontrado en el campo de la Didáctica Francesa a través de la teoría de las Situaciones Didácticas de Brousseau (1986), así como en la teoría de la Transposición Didáctica de Chevallard (1991). Ambas se basan en el estudio de las relaciones que se establecen entre el sistema didáctico (docente-alumno-saber). Una teoría más reciente es la Teoría de la Acción Conjunta (TACD) desarrollada por Sensevy (2011). La TACD toma de base estas dos anteriores teorías enfatizando además sobre la idea de que la enseñanza y el aprendizaje son acciones que se producen de una forma conjunta. En este sentido contempla que el estudio del aula debe ser realizado contemplando esta idea de conjunto. Para Sensevy (2011) la acción didáctica es una escena basada en la comunicación en la que las interacciones entre docente-alumnosaber son fundamentalmente transacciones originadas en un entorno muy concreto como es el aula. Una forma de estudiar el conjunto de transacciones producidas en una clase es a través del juego didáctico. El concepto de juego aporta permite la 
decodificación del aula en términos de acciones tanto del docente como del alumnado. Así el juego didáctico se define a través de dos descriptores: el medio y el contrato didácticos. El medio didáctico hace referencia al conjunto de elementos comunicativos, así como a la situación material, mientras que el contrato didáctico hace referencia a un contrato particular que se establece en un medio didáctico, el cual puede ser explícito o implícito. En la medida que se establece un nuevo contrato didáctico o un elemento del medio cambia, podemos considerar que se produce un cambio de juego. De esta forma, la TACD (Sensevy, 2011) permite una descripción sistemática de las situaciones didácticas facilitando la comprensión de la práctica docente.

\section{Objetivos}

Desde el papel clave que juega la formación inicial de maestros para provocar auténticos cambios en la enseñanza de las ciencias (Garriga Mercader et al., 2009), nos ha resultado de especial interés examinar cómo una estudiante del último curso del Grado de Magisterio aborda una actividad basada una metodología de indagación a un aula de Primaria. De esta forma, los objetivos que han regido el estudio han sido los siguientes:

1) Determinar aspectos generales de la sesión.

2) Examinar la estructura realizada por la futura maestra durante la actividad.

3) Examinar las estrategias de indagación manifestadas por los estudiantes de Primaria y su relación con los objetivos propuestos por la futura maestra.

\section{Métodos}

\section{Contexto y participantes}

El centro en cuestión es un centro con características especiales. Desde hace diez años existe una colaboración continuada con la universidad a través de diversos proyectos educativos del Gobierno Autonómico de la región. Tras años de trabajo en el diseño, puesta en marcha y elaboración de actividades, en la actualidad, las ciencias en este centro son trabajadas mediante actividades de indagación desde la etapa de Primaria e incluso desde la etapa de Infantil, desde hace varios años. Se trata así de un centro en el que el alumnado está acostumbrado a trabajar mediante el planteamiento de preguntas, realizando pequeñas investigaciones en grupo, razonando, argumentando y comunicando sus ideas al resto de la clase.

La clase analizada corresponde a un curso de $6^{\circ}$ de Primaria en la que una futura maestra realiza una actividad como parte de su Practicum. Se trata de un colegio público con un número de 17 estudiantes. La sesión se realiza con todo el grupo clase sin que los alumnos dispongan de libro de texto para su desarrollo. La actividad es videograbada para su posterior análisis.

\section{¿Cómo surge la actividad?}

En una sesión anterior con la futura maestra y a raíz de un dibujo elaborado por ella en la pizarra los alumnos se plantearon la duda sobre si sólo llovía en las montañas. El dibujo, al igual que la mayoría de las representaciones escolares sobre el ciclo del agua, representaba una nube que se había formado en el mar y que, trasladada por el 
viento, llovía sobre el pico de una montaña. Con el fin de desterrar esta idea, la futura maestra decidió elaborar una sencilla actividad a la que tituló: El experimento de los vasos de agua. El objetivo de la actividad consistió en que los alumnos dieran respuesta a las preguntas recogidas en la Tabla 2 . La selección de preguntas fue realizada por la futura maestra teniendo en cuenta ideas alternativas clásicas sobre el ciclo del agua (Marquez y i Plaza, 2007).

\begin{tabular}{|l|}
\hline \multicolumn{1}{|c|}{ Tabla 2. Preguntas de la actividad: El experimento de los vasos de agua. } \\
\hline \multicolumn{1}{|c|}{ Preguntas del experimento de los vasos de agua } \\
\hline 1. ¿Qué ha pasado en los vasos? \\
\hline 2. ¿Dónde está el agua que había antes? \\
\hline 3. ¿Siempre hay la misma cantidad de agua? \\
\hline 4. ¿Por qué creéis que en un vaso hay más agua líquida que en otro? \\
\hline 5. Sí pusiera un hielo encima del plástico, ¿qué pasaría? \\
\hline 6. ¿Pensáis que sólo llueve en las montañas? ¿Por qué? \\
\hline 7. ¿Cómo lo relacionaríais con este experimento? \\
\hline 8. Si pusiese los vasos igual, pero de noche ¿pasaría lo mismo? \\
\hline
\end{tabular}

\section{Instrumento}

Para el análisis de la videograbación se ha empleado la herramienta Transana (www.transana.org).

\section{Procedimiento y análisis de los datos}

\section{Escala macro (horas/minutos)}

Para la realización de las transcripciones se realizan varios visionados. Durante estos visionados se elabora una breve descripción de la sesión sobre los aspectos más relevantes como son los conceptos que se trabajan, la disposición de los alumnos o algunos de los elementos didácticos que están presentes en el aula. El nivel macro nos permite así dar cuenta de los principales aspectos de la sesión.

Escala meso (minutos)

En el nivel meso se lleva a cabo una estructuración de la sesión utilizando el concepto de juego didáctico (TACD; Sensevy, 2011). El juego didáctico se propone como una forma de identificar las distintas escenas/objetivos de aprendizaje que van sucediendo en una clase. La forma de identificar los juegos es a través de dos descriptores como son medio y el contrato didáctico o, en otras palabras, la regla que lo rige. Por ejemplo, cuando la maestra plantea la pregunta ¿Qué ha pasado en el vaso 1? la regla es observar aspectos que llamen la atención a los alumnos, hasta que de nuevo plantee otro objetivo con el que se entiende que la regla ha cambiado. Por otro lado, un cambio de medio didáctico sucede cuando por ejemplo la maestra no explicita un cambio de regla pero introduce un nuevo elemento (ej. un nuevo dispositivo, imagen, dato etc.) que no estaba presente hasta el momento. De esta manera, se considera un cambio de juego didáctico cuando se modifica el contrato o el medio didáctico (Sensevy, Tiberghien, Santini, Laubé y Griggs, 2008).

\section{Escala micro (minutos/segundos)}

En el nivel micro se realiza la codificación de las acciones del alumnado a través de un conjunto de categorías sobre las distintas estrategias de indagación (Milne, 2008) para alumnado de Primaria (Tabla 1). Para ello se tiene en cuenta la intencionalidad de los estudiantes, es decir, se codifica por ejemplo el periodo de tiempo en el que los alumnos buscan explicaciones a través de la categoría Buscar explicaciones al margen de si estas son científicamente válidas. Este nivel de análisis ofrece así una descripción detallada de las acciones de los estudiantes en torno a cada uno de los juegos didácticos planteados en la actividad (nivel meso). 


\section{Resultados}

\section{Escala macro: Aspectos generales de la sesión}

Los conceptos trabajados durante la actividad son los cambios de estado del agua así como aspectos parciales sobre sobre el ciclo del agua a escala hidro-geológica.

La sesión comienza con la futura maestra de pie mientras que los alumnos se hallan sentados en sus pupitres en grupos de cuatro. Previamente, la futura maestra dejó dos vasos de agua cubiertos ambos por un film de plástico. Uno de ellos se sitúa cerca de la ventana donde se encuentra el radiador (vaso 1). El otro vaso se sitúa en el lado opuesto del aula lejano a cualquier fuente de calor (vaso 2). Aunque los alumnos se disponen en los grupos habituales de trabajo, toda la sesión se dirige al gran grupo clase. Al comienzo de la actividad la futura maestra facilita en un folio a cada alumno las preguntas recogidas en la Tabla 2.

\section{Escala meso: Juegos didácticos}

Se han establecido un total de 17 juegos didácticos de duración variable (Tabla 3 ). Todos ellos, a excepción del 10, 15 y 16, guardan una relación directa con las preguntas que la futura maestra planificó (Tabla 2). De ello se deduce que los objetivos didácticos que se establecen durante la actividad quedan determinados por estas preguntas.

\begin{tabular}{|l|l|}
\hline \multicolumn{2}{|c|}{ Tabla 3. Relación de juegos didácticos establecidos y su duración temporal. } \\
\hline \multicolumn{1}{|c|}{ Juegos didácticos/Título } & Tiempo \\
\hline J1. ¿En qué va a consistir el experimento? & $(0: 00: 05-0: 01: 39)$ \\
\hline J2. ¿Qué ha pasado en el vaso? & $(0: 01: 39-0: 02: 31)$ \\
\hline J3. ¿Por qué hay gotitas en el plástico? & $(0: 02: 31-0: 04: 52)$ \\
\hline J4. ¿Hay la misma cantidad de agua? & $(0: 04: 52-0: 06: 27)$ \\
\hline J5. ¿Y si ponemos un hielo encima del plástico? & $(0: 06: 27-0: 12: 41)$ \\
\hline J6. ¿Sólo llueve en las montañas? & $(0: 12: 41-0: 19: 40)$ \\
\hline J7. ¿Por la noche ocurrirá lo mismo? & $(0: 19: 40-0: 22: 37)$ \\
\hline J8. ¿Qué ha pasado en el vaso? & $(0: 22: 37-0: 26: 49)$ \\
\hline J9. ¿Qué pasa si pones un hielo en el plástico? & $(0: 26: 49-0: 29: 12)$ \\
\hline J10. ¿Por qué llueve? & $(0: 29: 12-0: 33: 38)$ \\
\hline J11. ¿Dónde está el agua que había antes? & $(0: 33: 38-0: 35: 11)$ \\
\hline J12. ¿Por qué hay en un vaso más agua líquida que en el otro? & $(0: 35: 11-0: 36: 37)$ \\
\hline J13. ¿Sólo llueve en las montañas? & $(0: 36: 37-0: 38: 07)$ \\
\hline J14. ¿Cómo lo relacionarías con el experimento (lluvia)? & $(0: 38: 07-0: 50: 39)$ \\
\hline J15. ¿Por qué a veces llueve o nieva? & $(0: 50: 39-0: 55: 57)$ \\
\hline J16. ¿Siempre hay la misma cantidad de agua en las nubes? & $(0: 55: 57-0: 56: 28)$ \\
\hline J17. ¿Pasaría lo mismo por la noche? & $(0: 56: 28-1: 00: 03)$ \\
\hline
\end{tabular}

La actividad se estructura en dos partes claramente diferenciadas. La primera parte abarca hasta el juego didáctico 7 y se caracteriza por dar respuesta a cada una de las preguntas recogidas en la ficha (Tabla 2). Finalizado el juego 7 los alumnos redactan en grupo las respuestas. A continuación, comienza lo que se considera la segunda parte que abarca desde el juego 8 al juego 17, donde las respuestas son leídas en voz alta por los alumnos generando así un nuevo debate. Esta especie de 'repaso' produce una aparente repetición de los juegos didácticos.

Todos los juegos, a excepción del juego 15 y 16, poseen como contrato didáctico alguna de las preguntas que aparecen en la ficha (Tabla 2) siendo todos ellos introducidos y gestionados por la futura maestra. Únicamente los juegos 15 y 16 (¿Por qué a veces llueve o nieva? y ¿Siempre hay la misma cantidad de agua en las nubes?) no guardan una relación con alguna de las preguntas de la ficha. Ambos juegos se caracterizan por regirse por una pregunta que ha sido planteada por una alumna cada uno de ellos. El hecho de que al final de la sesión sean dos alumnas las que acaben planteando preguntas susceptibles de ser debatidas es considerado como 
un dato muy positivo desde el punto de vista de la indagación (García González y Furman, 2014).

Se percibe así que el tipo de estructura planteada por la futura maestra se relaciona con secuencias de aprendizaje basada en el uso de preguntas. En especial un papel importante juega las preguntas que la futura maestra planificó con el objetivo de que los alumnos apreciasen los movimientos del agua a través de los cambios de estado.

\section{Escala micro: Estrategias de indagación desarrolladas por los alumnos de Primaria}

De la codificación de las estrategias de indagación que son percibidas en el alumnado durante el escrutinio de la videograbación se ha realizado la Figura 2. Esta Figura recoge de forma visual el conjunto de estrategias que desarrolla el alumnado en cada uno de los juegos didácticos. Así, por ejemplo, podemos leer que en el juego 1 se ha detectado el desarrollo de las estrategias: (1) Explorar (2) Observar del fenómeno (3) Identificar evidencias.

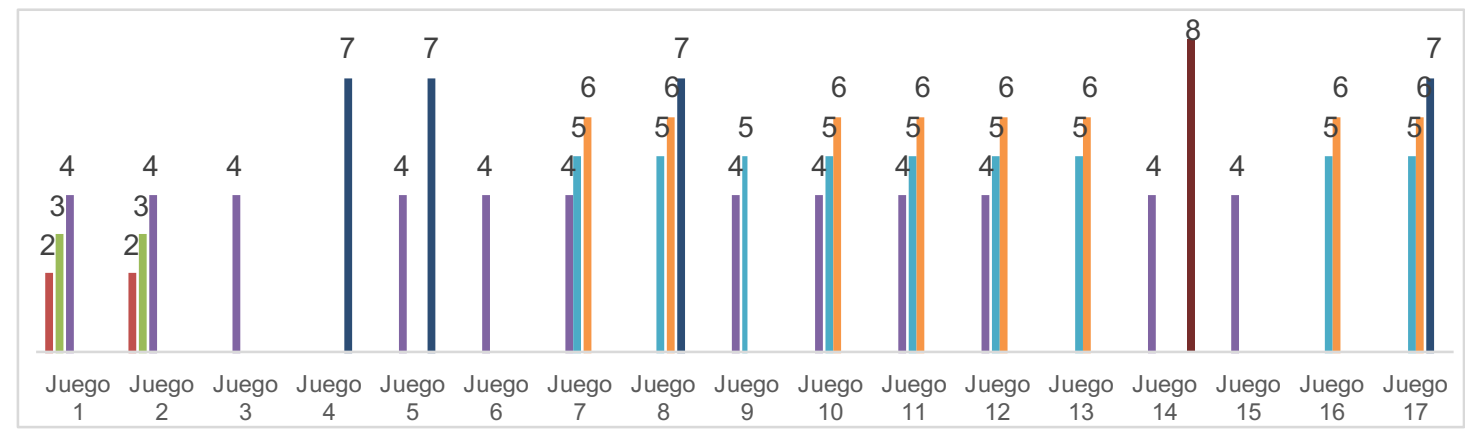

Figura 1. Representación de las estrategias didácticas desarrolladas por los alumnos durante la secuencia temporal de juegos didácticos. Eje x: Secuencia temporal de los juegos didácticos. Eje y: (1) Explorar; (2) Observar del fenómeno; (3) Identificar evidencias; (4) Buscar explicaciones; (5) Comprobar la explicación/ Evaluar; (6) Síntesis de conocimiento; (7) Predicción/Más investigaciones; (8) Aplicación a otros contextos

De la Figura 1 se deduce que los estudiantes de Primaria son capaces de desarrollar todas las estrategias de indagación a lo largo de toda la actividad (Figura 1). Llama la atención cómo la estrategia (4) Buscar explicaciones está presente en todos los juegos didácticos, de manera que los estudiantes parecen no cesar en reelaborar y refinar sus explicaciones. Se puede decir que a medida que avanza la actividad los alumnos son capaces de producir todas las estrategias propias de la indagación, desde los niveles más iniciales de la indagación hasta niveles superiores. Entre los juegos 1 y 4 se aprecia una evolución de estrategias como es la secuencia (1) Explorar, (2) Observar del fenómeno, (3) Identificar evidencias y (4) Buscar explicaciones. Posteriormente, en el juego 5, surge la estrategia de (7) Predicción/Más investigaciones, lo que produce un aparente 'salto' que va de la búsqueda de explicaciones a la realización de predicciones con ese conocimiento. Una explicación la encontramos teniendo en cuenta el contrato didáctico establecido en el juego 5 el cuál demanda directamente la realización de predicciones sobre el experimento a los alumnos.

Es a partir del juego 7 cuando los alumnos comienzan a producir estrategias de mayor complejidad como son: (4) Buscar explicaciones, (5) Comprobar la explicación/ Evaluar y (6) Síntesis de conocimiento. Recordemos que es a partir del juego 7 cuando la futura maestra solicita de nuevo a los alumnos que respondan las preguntas recogidas en la ficha. En este sentido, parece ser que la aparente repetición de los 
juegos didácticos ofrece a los estudiantes la capacidad de desarrollar estrategias de indagación con una mayor complejidad.

En cuanto a la estrategia (7) Predicción/Más investigaciones ésta aparece principalmente en aquellos juegos cuyo contrato insta al alumnado a realizar predicciones. Encontramos, por tanto, una relación directa entre la demanda realizada a los alumnos de forma formal a través de una pregunta y la estrategia de resolución empleada para dar respuesta a esa demanda.

En los últimos juegos, del 8 en adelante, los estudiantes continúan refinando sus explicaciones sometiéndolas a evaluación, recapitulando y sintetizando las ideas, motivo por el cual se han codificado secuencias de estrategias del tipo (4) Buscar explicaciones, (5) Comprobar la explicación/ Evaluar, (6) Síntesis de conocimiento. Cabe resaltar que en el juego 10 (¿Por qué llueve?) la futura maestra sugiere a los alumnos que expliquen el fenómeno de la lluvia relacionándolo con las observaciones realizadas previamente. Sería esperable que los estudiantes desarrollasen la estrategia (8) Aplicación a otros contextos no obstante, dicha estrategia no es manifestada. La escasa presencia de esta estrategia durante la actividad pudiera interpretarse como una de las mayores dificultades encontrada por estos alumnos, la de trasladar el conocimiento generado sobre los cambios de estado al contexto atmosférico.

\section{Conclusiones}

Este estudio se centra en el análisis de una actividad de indagación llevada a cabo por una estudiante de Magisterio en un aula de Primaria durante su Practicum universitario. El estudio ha mostrado cómo un análisis del aula a través del concepto de juego didáctico (TACD; Sensevy 2011) permite una descripción detallada sobre la estructura de una sesión en relación con los objetivos de aprendizaje. Por otro lado, gracias al sistema de categorías sobre estrategias de indagación, basado en el trabajo de Milne (2008), ha sido posible una categorización minuciosa sobre las acciones de los alumnos de Primaria. En este sentido, queremos destacar la pertinencia de la metodología empleada para poder analizar a varias escalas qué ocurre cuando se trata de aprender en el aula (Tiberghien y Malkoun, 2010).

En esta sesión, la futura maestra llevo a cabo una estructuración de la actividad basada en dos partes claramente diferenciadas. En la primera parte la futura maestra empleó un conjunto de preguntas que había planificado para desarrollar los juegos didácticos. Cada juego tenía como objetivo dar respuesta a cada una de estas preguntas. En la segunda parte de la sesión, y tras el debate generado con los estudiantes, volvió a revisar las mismas preguntas con los estudiantes. Así el conocimiento es sometido a una evaluación por el gran grupo clase de igual modo que ocurre en la ciencia donde el conocimiento es sometido a evaluación.

Un dato revelador lo encontramos al final de la sesión cuando dos alumnas plantean dos preguntas que desencadenan dos pequeños debates. Estas preguntas son: ¿Por qué a veces llueve o nieva? y ¿Siempre hay la misma cantidad de agua en las nubes? En ambos casos la futura maestra permite que fueran los alumnos los que pensasen y aportasen razones para dar respuesta, dato considerado muy positivo desde el punto de vista de la indagación (García González y Furman, 2014).

Por otro lado, los resultados muestran cómo la naturaleza de los datos, teórica o experimental, o el tipo de estructuración de la actividad que realiza la futura maestra se convierten en aspectos claves para el desarrollo de estrategias de indagación. Los 
datos de tipo experimental son imprescindibles para llevar a cabo estrategias como son la observación o la identificación de evidencias, que de otra manera deben ser referidas (por ejemplo, cuando hablamos de que los cristales se empañan). El desarrollo de estrategias de indagación en el alumnado parece tener cierto éxito en la medida que los estudiantes de Primaria presentan indicios sobre un trabajo de las ciencias basado en un constante razonamiento, interactuando con el medio y con sus marcos teóricos previos, para finalmente comunicar ideas que poco a poco van refinando oralmente.

\section{Discusión}

En este estudio se concluye cómo: 1) la claridad de los objetivos planteados en forma de preguntas por parte de la futura maestra, 2) el tipo de estructuración de la sesión en la que el conocimiento es sometido a evaluación, así como 3) las estrategias manifestadas por los estudiantes, permite calificar la actividad como un proceso de indagación guiada (Banchi y Bell, 2008). No obstante, cabe señalar la particularidad del centro, siendo un centro atípico en la medida que desde la etapa de Infantil los alumnos de Primaria comienzan a trabajar mediante actividades indagatorias. Este hecho pone de manifiesto que al margen de la guía realizada por la futura maestra, para la construcción de rutinas de aprendizaje se requiere la colaboración de todo el profesorado y a lo largo de toda la etapa educativa. A través de estas pequeñas actividades en la etapa del Practicum se posibilita la implantación paulatina de trabajos por indagación en las escuelas, donde tanto maestros en activo como futuros docentes se enfrentan a nuevos entornos de aprendizaje. También queremos destacar la planificación de estas actividades como una forma en la que los futuros maestros tomen conciencia sobre los contenidos y objetivos que pretenden tratar, y adecuarlos en forma de preguntas. Además, esta planificación previa revierte en una confianza sobre el maestro, de manera que al haber dedicado un tiempo a su diseño y puesta en práctica la sensación de eficacia y autoconfianza mejora (Crujeiras-Pérez, 2017).

\section{Referencias}

Abd-El-Khalick, F., \& Akerson, V.L. (2004). Learning about nature of science as conceptual change: Factors that mediate the development of preservice elementary teachers' views of nature of science. Science Education, 88(5), 785-810.

Banchi, H., \& Bell, R. (2008). The Many Levels of Inquiry. Science and Children, 46(2), 26-29.

Brousseau, G. (1986). Fondements et méthodes de la didactiques des mathématiques. Recherches en Didactique des Mathématiques, 7(2), 33-115.

Chevallard, Y. (1991). La transposición didáctica: del saber sabio al saber enseñado, Buenos Aires: Aique.

Crujeiras Pérez, B., \& Jiménez Aleixandre, M. J. (2015). Desafíos planteados por las actividades abiertas de indagación en el laboratorio: articulación de conocimientos teóricos y prácticos en las prácticas científicas, Enseñanza de las Ciencias, 33(1), 63-84.

Crujeiras-Pérez, B. (2017). Análisis de las estrategias de apoyo elaboradas por futuros docentes de educación secundaria para guiar al alumnado en la indagación, Revista Eureka sobre Enseñanza y Divulgación de las Ciencias, 14(2), 473486.
Crujeiras-Pérez, B., \& Cambeiro, F. (2018) Una experiencia de indagación cooperativa para aprender ciencias en educación secundaria participando en las prácticas científicas, Revista Eureka sobre Enseñanza y Divulgación de las Ciencias, 15(1), 1201.

Driver, R., Guesne, E., \& Tiberghien, A. (1985). Children's ideas in Science. Glasgow: Milton Keynes, Open University Press.

Duschl, R.A. (1995). Más allá del conocimiento: los desafíos epistemológicos y sociales de la enseñanza mediante el cambio conceptual, Enseñanza de las Ciencias, 13(1), 3-14.

Flick, L., \& Lederman, NG. (Eds.) (2004). Scientific inquiry and nature of science: implications for teaching, learning, and teacher education. Dordrecht, The Netherlands: Kluwer Academic Publishers.

García González, S. M., \& Furman, M. G. (2014). Categorización de preguntas formuladas antes y después de la enseñanza por indagación. Praxis \& saber, 5(10), 75-91.

García González, S., \& Furman, M. (2014). Categorización de preguntas formuladas antes y después de la enseñanza por indagación. Praxis \& Saber, 5(10), 75-91. 
Garriga Mercader, L., Langreo Valverde, S., Martínez Aznar, M. M., Moreno González, F., Ochoa Cañigueral, L., \& Ribas Niñez, C. (2009). Educación científica 'Ahora': el informe Rocard. Ministerio de Educación.

Gil Pérez, D. (1991). ¿Qué hemos de saber y saber hacer los profesores de ciencias? Enseñanza de las Ciencias, 9(1), 6977.

Kidron, I., Bikner-Ahsbahs, A., Monaghan, J., Radford, L., \& Sensevy, G. (2012). CERME7 Working Group 16: Different theoretical perspectives and approaches in research in mathematics education, Research in Mathematics Education, 14(2), 213-214.

Kuhn, T.S. (1986). La estructura de las revoluciones cientificas. Madrid, Fondo de Cultura Económica.

Márquez, C., \& i Plaza, J. B. (2007). Una propuesta de análisis de las representaciones de los alumnos sobre el ciclo del agua. Enseñanza de las Ciencias de la Tierra, 15(3), 280-286.

Martínez-Chico, M., Jiménez Liso, M.R., \& Lucio-Villegas, R.L.G. (2014). Efecto de un programa formativo para enseñar ciencias por indagación basada en modelos, en las concepciones didácticas de los futuros maestros, Revista Eureka sobre Enseñanza y Divulgación de las Ciencias, 12(1), 149-166.

Milne, I. (2008). Creative exploration: Doing science in a primary school context. In R. Lakeside, R. Fitzgerald \& T. W. Nielsen (Eds), Proceedings of the Sixth International Conference on Imagination and Education, 48-57. Australia: Camberra, University of Camberra.

National Research Council (2007). Taking Science to School: Learning and Teaching Science in Grades K-8. Washington, DC: The National Academies Press.

Quílez, M.J.G., Peña, M.B.M., de la Gándara Gómez, M., Hernández, J. M. C., \& Gracia, A. L.C. (2008). De la universidad a la escuela: no es fácil la indagación científica, Revista Interuniversitaria de Formación del Profesorado, 63, 81-100.

Rivero García, A., Solís Ramírez, E., Porlán Ariza, R., Azcárate Goded, M. D.P., \& Martín del Pozo, R. (2017). Cambio del conocimiento sobre la enseñanza de las ciencias de futuros maestros, Enseñanza de las Ciencias, 35(1), 29-52.

Sensevy, G. (2011). Le sens du savoir. Éléments pour une théorie de l'action conjointe en didactique. Bruxelles: de Boeck.

Sensevy, G., Tiberghien, A., Santini, J., Laubé, S., \& Griggs, P. (2008). An epistemological approach to modeling: Cases studies and implications for science teaching. Science education, 92(3), 424-446.

Solé Llussà, A., Aguilar Camaño, D., Ibáñez, M., \& Coiduras Rodríguez, J. L. (2018). Análisis de la comunicación de experiencias indagadoras presentadas en congresos de ciencias dirigidos a alumnos de educación infantil y primaria, Revista Eureka sobre Enseñanza y Divulgación de las Ciencias, 15(1), 1302.

Terigi, F., \& Diker, G. (1997). La formación de maestros y profesores: hoja de ruta. Buenos Aires. Editorial Paidós.
Tiberghien, A., \& Malkoun, L. (2010). Analysis of classroom practices from the knowledge point of view: how to characterize them and relate them to students' performances. Revista Brasileira de Pesquisa em Educação em Ciências, 10(1), 1-32.

Vilchez González J.M., \& Bravo Torija B. (2015). Percepción del profesorado de ciencias de educación primaria en formación acerca de las etapas y acciones necesarias para realizar una indagación escolar, Enseñanza de las Ciencias, 33(1), 185202.

Windschitl, M., Thompson, J., \& Braaten, M. (2008). Beyond the scientific method: Model based inquiry as a new paradigm of preference for school science investigations, Science education, 92(5), 941-967. 